\title{
Pre-implementation guidelines for infectious disease point-of-care testing in medical institutions
}

\author{
Annemiek A van der Eijk', Andrei N Tintu² \& John P Hays*,3
}

Infectious disease point-of-care test (ID-POCT) devices are becoming widely available, and in this respect, international quality standards and guidelines are available for consultation once ID-POCT has been implemented into medical institutions. However, specific guidelines for consultation during the initial pre-implementation decision-making process are currently lacking. Further, there exist pre-implementation issues specific to ID-POCT. Here we present pre-implementation guidelines for consultation when considering the implementation of ID-POCT in medical institutions.

First draft submitted: 16 June 2016; Accepted for publication: 14 September 2016;

Published onilne: 6 December 2016

Point-of-care test (POCT) devices are widely available for use in medical institutions [1] and in this respect, international quality standards and guidelines are available regarding their implementation [2], for example: ISO 15189:2012 - 'Requirements for Quality and Competence' [3]; ISO22870:2006 'Point-of-Care Testing - Requirements for Quality and Competence' [4]; ID-POCT01-A2 'Point-of-Care Connectivity' - Approved Standard-Second Edition [5] and 'Diagnosing Infection at the Point of Care' [6] etc. However, the implementation of dedicated infectious disease POCT (ID-POCT) devices into medical institutions tends to lag behind that of POCT devices used in many other medical disciplines, for example, clinical chemistry. Further, though many of the implementation issues for POCT and ID-POCT are similar (see references above), there still exist crucial pre-implementation differences between POCT and ID-POCT before their implementation into medical institutions [7-9].

One of the hurdles to implementing ID-POCT is a lack of specific ID-POCT guidelines and solutions which can be used to guide ID-POCT stakeholders, for example, physicians, laboratory managers, ID-POCT manufacturers and retailers, financial personnel etc. in their decision making before actual implementation issues are decided. Further, there exist specific ID-POCT pre-implementation issues which may not be applicable to other forms of POCT devices.

In this Perspective article, the authors present a simple and 'holistic' set of ID-POCT guidelines that can be used by ID-POCT stakeholders to guide their pre-implementation ID-POCT decision-making processes within their own medical institutions. In this respect, 'holistic' means taking into account the whole range of stakeholders involved in the implementation of ID-POCT within medical institutions, including clinicians, diagnosticians, laboratory managers, healthcare financiers, manufacturers of ID-POCT and patients (specifically the actual clinical needs of the

'Department of Viroscience, Erasmus University Medical Center Rotterdam (Erasmus MC), Rotterdam, the Netherlands

2Department of Clinical Chemistry, Erasmus University Medical Center Rotterdam (Erasmus MC), Rotterdam, the Netherlands

${ }^{3}$ Department of Medical Microbiology \& Infectious Diseases, Erasmus University Medical Center Rotterdam (Erasmus MC), Rotterdam,

the Netherlands

*Author for correspondence: Tel.: +31 31107032 17; j.hays@erasmusmc.nl

\section{KEYWORDS}

- guidelines $\bullet$ infectious diseases $\bullet$ point-of-care testing 


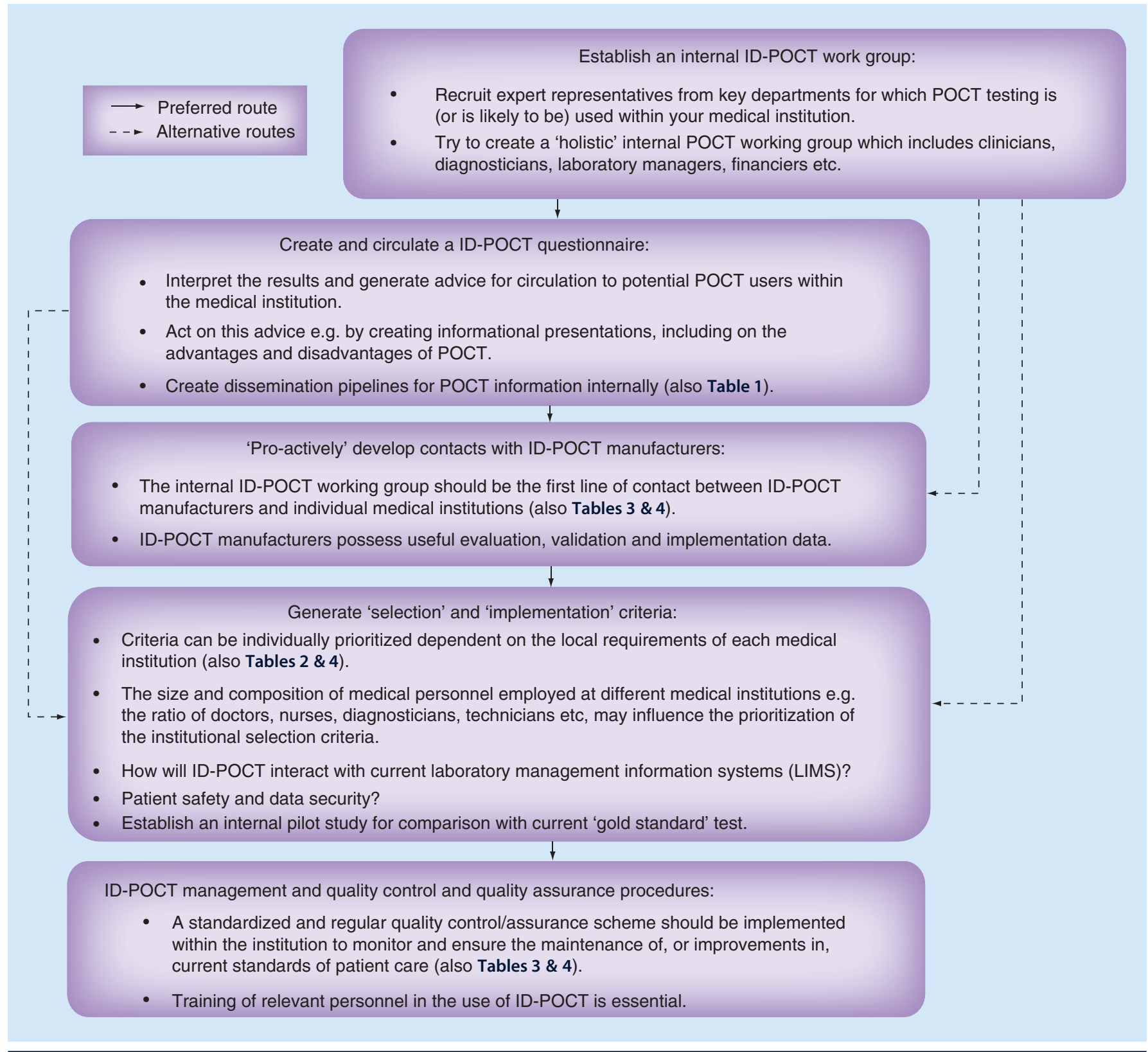

Figure 1. Pre-implementation planning for infectious disease point-of-care testing in medical institutions.

patient). By including all of these stakeholders in the decision-making process, informed and accurate decisions can be made regarding the needs and usefulness of ID-POCT within individual medical institutions. These guidelines are based on the experiences of a recently established point-of-care expert group at the Erasmus University Medical Center Rotterdam (Erasmus MC), a tertiary care university medical center based in Rotterdam, the Netherlands, with approximately 1,220 hospital beds, and which receives approximately 40,000 in-patients and
500,000 out-patient visits per year. The guidelines will be useful to both medical institutions and ID-POCT kit manufacturers and encourage these organizations (with potentially conflicting goals) to better understand and interact with one another in order to achieve the successful implementation of ID-POCT into existing analytical workflows within medical institutions. Although the intention of this article is to present a 'universal' set of guidelines, it should be noted that not all of the suggestions made may be applicable to all healthcare providers in all countries and 
adaptation of the guidelines may be necessary in order to meet with local or national requirements.

An 'overview of the ID-POCT pre-implementation process' per se is shown in Figure 1, including several 'alternative routes' that may be taken dependent on individual circumstances within different medical institutions. For the implementation of ID-POCT on a small scale i.e. institutions with only one or two medical departments, creating and circulating an ID-POCT questionnaire may not be necessary as the opinions of end-users can be simply obtained via face-to-face discussions. However, this particular alternative route is not recommended for larger medical institutions, or where there is a debate regarding which particular ID-POCT device is most suitable for implementation. Similarly, it is not absolutely necessary to develop contacts with ID-POCT manufacturers for the small-scale use of ID-POCT. However, ID-POCT manufacturers can be a good source of rapid information regarding new developments for their particular ID-POCT device, and if large quantities of consumables will be required for a particular
ID-POCT, then it may be possible to negotiate financial discounts, especially if there is the possibility of competition between two or more ID-POCT companies for your business.

Tables 1-3 and Box 1 provide more detail to Figure 1 and group the guidelines into general themes relating to: suggested 'dissemination pipelines' (Table 1) - essentially, knowledge gathering and knowledge dissemination guidelines in order to understand and react to the needs and perceptions of ID-POCT stakeholders, thereby providing a broad base on which to build ID-POCT implementation discussions; potential 'selection criteria' (Box 1) - considerations which may be relevant to ID-POCT stakeholders in different medical institutions; 'pro-active' interactions (Table 2) - generating institution-specific ID-POCT purchase and implementation guidelines; and ID-POCTspecific pre-implementation issues (Table 3) - there are several ID-POCT preimplementation issues which are particularly important for certain types of ID-POCT devices and situations.

Consultation and/or adoption of these guidelines will establish a firm foundation for the

Table 1. Suggested 'dissemination pipelines' for distributing institutional infectious disease point-of-care test information (both internally and externally).

\begin{tabular}{|c|c|}
\hline Pipeline & Comments \\
\hline Institution-wide 'intranet site' and or 'service portal' & $\begin{array}{l}\text { What potentially appropriate ID-POCT are available within and } \\
\text { outside the medical center? } \\
\text { - Keep up-to-date with new advances in the market } \\
\text { Create a dedicated institutional ID-POCT website where colleagues } \\
\text { can obtain ID-POCT information when they need it (a simple } \\
\text { website could be created with links to external sources of } \\
\text { information [10]) } \\
\text { - Include these guidelines, implementation costs, quality control } \\
\text { issues, etc } \\
\text { - Create an internal ID-POCT discussion forum }\end{array}$ \\
\hline Individual lectures and face-to-face meetings & $\begin{array}{l}\text { Generate new ID-POCT learning materials including e-learning } \\
\text { modules and self-study materials }\end{array}$ \\
\hline Online & $\begin{array}{l}\text { Communicate your medical institutions experiences with ID-POCT via } \\
\text { Youtube, Facebook or Twitter }\end{array}$ \\
\hline Conferences and scientific publications & $\begin{array}{l}\text { - Present your implementation experiences at scientific conferences } \\
\text { - Publish the results of your institutions ID-POCT questionnaire (if } \\
\text { applicable) } \\
\text { - Publish your own findings regarding quality of ID-POCT devices, for } \\
\text { example, sensitivity, specificity etc }\end{array}$ \\
\hline $\begin{array}{l}\text { Determine consequences of ID-POCT testing on current and future } \\
\text { research and education activities (applicable to medical institutions } \\
\text { with academic responsibilities) }\end{array}$ & $\begin{array}{l}\text { Effect of ID-POCT on the maintenance of up-to-date and state-of-the- } \\
\text { art teaching and training programs in the rapidly changing field of } \\
\text { medical diagnostics }\end{array}$ \\
\hline $\begin{array}{l}\text { Prepare standard communication texts - leaflets etc. - informing } \\
\text { patients of the advantages and disadvantages of using (a specific) } \\
\text { ID-POCT }\end{array}$ & $\begin{array}{l}\text { Patients should be considered as stakeholders and therefore } \\
\text { informed over the reasons for implementing ID-POCT (particularly in } \\
\text { comparison with current 'gold standard' laboratory-based techniques) }\end{array}$ \\
\hline
\end{tabular}


Table 2. 'Pro-active' interactions available to medical institutions and infectious disease point-of-care test manufacturers to help them with infectious disease point-of-care test implementation.

Interaction ID-POCT manufacturer/developer working together Comments

with medical institutions

Make and maintain contacts with institutional ID-POCT working

groups

Understand the key institutional workflows and current gold

standard testing criteria

Identify where a particular ID-POCT test assay would bring

advantages over existing institutional workflows and report this to

the relevant ID-POCT working group

Keep the ID-POCT working group up to date on new developments

within the fields of individual ID-POCT test assays

Provide details of relevant scientific publications to institutional ID-

POCT working groups and end-users

Provide details of relevant medical institutions where the ID-POCT

has already been successfully established

Identify institutional ID-POCT test assay selection criteria at an early

stage and develop the ID-POCT test assay to take into account these

institutional needs

Identify institutional ID-POCT test assay selection criteria at an early

stage and develop a business model based on these criteria

Keep up-to-date with the results of ID-POCT funding awards and

opportunities

ID-POCT: Infectious disease point-of-care test
Making contacts with individual end-users, for example, heads of departments, may not be sufficient in helping implement ID-POCT test assays in medical institutions

Use the ID-POCT working group and individual end-users as a source of advice regarding individual institutional workflows

Possible advantages include: increased specificity, increased sensitivity, decreased costs, decreased time to result, increased throughput, ease of use, etc

How is/has the ID-POCT test assay being/been developed/updated to meet the specific requirements of individual medical institutions?

What scientific evidence is available regarding the test characteristics of the ID-POCT test assay?

Be aware that individual workflows may vary between different medical institutions, so 'one size may not fit all'

Try to develop ID-POCT test assays which meet as many selection criteria as possible

Try to develop a business model which can successfully compete with current (and future) financial criteria managed by medical institutions Current examples include the 'Longitude Prize' [11] and the 'Better Use of Antibiotics' Prize [12] successful implementation of ID-POCT within many different types of medical institutions. Further, ID-POCT assay manufacturers will receive the opportunity to pro-actively inform and interact at all stages within the ID-POCT implementation process. However, it should be noted that such interactions should be mutually beneficial and ideally based on solid scientific evidence or previous implementations of ID-POCT assays into the medical environment, rather than simply being a 'lobbying' or 'sales' exercise by individual ID-POCT assay manufacturers/developers to individual medical institutions. The large scale successful implementation of ID-POCT in medical institutions could ultimately be beneficial for all ID-POCT stakeholders, including clinicians, diagnosticians, laboratory managers, healthcare providers and (most importantly) patients themselves.

Finally, it should be noted that the main emphasis of these guidelines has been directed toward the implementation of ID-POCT within medical institutions per se, rather than the development of guidelines for the use of (disposable) ID-POCT in: 'low experience' environments, for example, patients at home; 'extreme' environments, for example, battlefield situations, astronauts in space etc. or 'limited resource' environments, for example, in developing countries during infectious disease epidemics (Ebola, Zika viruses, Enterohemorrhagic Escherichia coli food-borne disease outbreaks etc.) where medical personnel may be at special risk of (nosocomial) infection or staff shortages may occur. However, this does not mean that the above guidelines are invalid for these situations, rather that individual guidelines may have to be adapted, ignored or some guidelines given more 'weight' than other guidelines in order to best serve the requirements of the individual situation, for example, user education, urgency of the situation; explosive increase in ID-POCT throughput requirements, ID-POCT component stability, ID-POCT costs etc. Ultimately, it is the choice of the (end) user who will determine which guidelines are useful for their own particular situation.

\section{Conclusion}

POCT has the potential to transform current healthcare practices. However, (pre-)implementation issues may be limiting the introduction and successful use of POCT within many medical environments and institutions. This 
Box 1. List of potential 'selection criteria' for consideration prior to infectious disease point-of-care test implementation.

\section{Technology}

- The extent to which the technology:

- Is valuable and affordable

- Has been evaluated - including in the scientific literature - with respect to specimen collection, specificity, sensitivity, reproducibility, cost per test etc

- Fits within the existing IT infrastructure (e.g., security, bandwidth)

- Requires certification (appropriate for regional, national or international regulations where the ID-POCT will be performed)

- Is applicable to the work environment, for example, hospital general wards, emergency rooms, family doctors' offices

\section{Implementation}

- Is the test approved for use by (inter)national regulatory authorities?

- The simplicity of complying with existing laws and regulations

- The extent to which the new service can be incorporated into existing workflows

- Are the appropriate resources available (including extra laboratory capacity)?

- The degree to which the risks associated with implementation can be controlled

- The speed at which the new ID-POCT test service can be launched

- Cultural barriers - perceived lack of confidentiality and individual/societal stigma associated with the result, for example, HIV rapid tests

\section{Use and alternatives}

- The degree of acceptance by medical institution care providers and customers/clients for this kind of service

- The number of clients who would want/can/should use the new ID-POCT test service

- The growth expectation for the ID-POCT test new service

- Are there alternatives to the new ID-POCT test service available?

\section{Operation}

- Interconnectivity of ID-POCT with existing labratory information management systems for your institution.

- Simplicity in learning to use the new ID-POCT technology

- Availability of training courses for doctors, nurses, technicians etc. and planning for regular (re-)accreditation/(re-)certification

- Are the consumable materials required for the new ID-POCT test service available?

- The ease by which ID-POCT problems can be solved by medical institution personnel, for example, self-repairs

Costs

- The extent of funding for the development of the ID-POCT test service in question

- Cost of replacing existing laboratory equipment (if applicable)

- Cost of training and maintaining ID-POCT user expertise

- Need for additional, or frequency, of quality control/quality assurance programs

- Is reimbursement available for ID-POCT use (insurance companies or healthcare providers)?

- The price of ID-POCT fits with economic and financial restrictions, for example, low- and middle-income countries?

- The time required before the investment is recouped

- The financial risks can be controlled

Strategy

- The extent by which the new ID-POCT test service:

- Encourages health-related participation with society

- Enhances the independence of patients

- Supports efficiency in the care process

- Contributes to a positive image of the medical environment

D-POCT: Infectious disease point-of-care test.

is especially true for ID-POCT. Therefore, in this publication, the authors provide preimplementation guidelines for consideration by ID-POCT stakeholders, including healthcare workers, laboratory managers, financiers and POCT developers, when deciding on the possible implementation of ID-POCT within medical institutions.

\section{Future perspective}

The implementation of ID-POCT offers much potential, but currently lags behind the 


\section{Table 3. ID-POCT Specific Pre-Implementation Issues.}

\section{Specific ID-POCT Issue}

ID-POCT generally detect multiple specimen targets during the testing of a single

\section{Comments}

- Should the clinician ignore the results from targets that have not been requested?

- What are the legal and moral issues associated with ignoring test results obtained (but not requested) by the clinician?

- Are healthcare providers willing to pay for the costs of these extra tests (compared to the use of a single target-specific ID-POCT), even if these are not requested by the clinician?

Quality control and quality assurance of ID-POCT multiple target detection devices is more complex than single target POCT

- How often should each target be QC/QA? If an ID-POCT can detect 20 targets simultaneously, should all 20 targets receive QC/QA at the same time? If a target only receives QC/QA once every 3 months, instead of for example once every month, then many more patients may have to be informed that their test results were incorrect if that 3 monthly test fails. This could lead to higher morbidity and mortality compared to a ID-POCT which receives QC/QA every week or month

- What are the extra costs involved in the quality control/quality assurance of multi-target ID-POCT?

- Is there a reliable supplier of reference material for each of the targets in a multi-target ID-POCT?

- Will healthcare providers be willing to pay for additional QC/QA testing for rare, or unused targets?

- Sample collection and storage issues should be discussed, agreed and implemented in order to provide a biobank of duplicate patient samples in case quality control procedures indicate that the ID-POCT has failed

Potential use of emergency legislation in times of biological crises

Be aware of emergency legal issues, for example, the US FDA EUA. "Under section 564 of the Federal Food, Drug, and Cosmetic Act (FD\&C Act), the FDA Commissioner may allow unapproved medical products or unapproved uses of approved medical products to be used in an emergency to diagnose, treat, or prevent serious or life-threatening diseases or conditions caused by chemical, biological, radiological and nuclear defence threat agents, when there are no adequate, approved, and available alternatives" $[13,14]$

Provision of personal protective equipment, high risk testing, and isolation units

Access to reference material The explosive spread of highly infectious agents e.g. Zika, Ebola, MERS-CoV, may require the rapid implementation of ID-POCT. Suitable protocols, training and equipment need to be available in order to ensure the success of the rapid implementation of ID-POCT, especially if these tests do not form part of the 'normal' diagnostic packet of medical institutions

Currently, specimens sent to microbiology laboratories for the detection of infectious disease pathogens provide an extremely useful resource for epidemiological studies, as well as being a source of novel microbial pathogens. If ID-POCT becomes widely used in hospital wards, at family doctor practices, or even at home, what will the effect be on the successful tracking and surveillance of microbial pathogens? Think for example about the yearly production of an effective influenza vaccine, or the efficient monitoring of carbapenem antibiotic resistance in Europe

Universal primers In order to provide broad coverage for the detection of a specific microbial pathogen, ID-POCT tend to use primers directed against universal sequences that are present within all known members of a particular microbial species. This means that there will be much less information available regarding the epidemiology of specific microbial strains compared with, for example a 'catch-all' technique such as bacterial culture (where bacteria can be cultured and their specific epidemiological profiles determined by further processing e.g. gene sequencing)

Next generation sequencing IDPOCT
- One exception to the 'access to reference material' and 'universal primers' comments mentioned above, is the use of next-generation sequencing-based ID-POCT, which could provide specific epidemiological information on the target pathogen detected. However, there may be potentially multiple pathogens present within a clinical sample and the experience of clinicians may be required in order to determine which potential pathogen is associated with the current disease state

- Next-generation sequencing ID-POCT may require intensive bioinformatics and software support, which means that this kind of test may not be (currently) suitable for applications outside of secondary or tertiary care medical institutions. Additionally, algorithms will need to be developed which simulate the 'experience of clinicians' when making decisions regarding the relative contribution of a potential pathogen to the current disease state 
POCT advances associated with other clinical disciplines. The successful implementation of ID-POCT in the future will be characterized by: a shift in emphasis from 'technologically based' to 'clinically based' ID-POCT solutions; a general acceptance of ID-POCT by the medical profession (most likely led by an increase in awareness of the advantages of ID-POCT at family doctor practices, in emergency rooms and in the home environment); the development of scientific/manufacturing pipelines; and the adoption of ID-POCT for monitoring and control of new diseases and (exotic) disease outbreaks. However, one of the main remaining obstacles to the implementation of ID-POCT will still be its financial reimbursement.
Financial \& competing interests disclosure

This publication was supported by a Horizon 2020

European Union grant under grant agreement GA-633780 ("DIAGORAS" - www.diagnoras.eu). The authors have no other relevant affiliations or financial involvement with any organization or entity with a financial interest in or financial conflict with the subject matter or materials discussed in the manuscript apart from those disclosed.

No writing assistance was utilized in the production of this manuscript.

\section{Open access}

This work is licensed under the AttributionNonCommercial-NoDerivatives 4.0 Unported License. To view a copy of this license, visit http://creativecommons.org/ licenses/by-nc-nd/4.0/

\section{EXECUTIVE SUMMARY}

- The implementation of dedicated infectious disease point-of-care test (ID-POCT) devices into medical institutions tends to lag behind that of POCT devices used in many other medical disciplines.

- There exist crucial preimplementation differences between POCT and ID-POCT before their implementation into medical institutions.

- The authors present a simple, but 'holistic' set of ID-POCT guidelines that can be used by ID-POCT stakeholders to guide their preimplementation ID-POCT decision-making processes.

- 'Dissemination pipelines' provides knowledge gathering and dissemination guidelines in order to understand and react to the needs and perceptions of ID-POCT.

- Potential 'selection criteria' considers the selection criteria which may be relevant to ID-POCT stakeholders in different medical institutions.

- 'Pro-active' interactions describes how medical institutions and ID-POCT manufacturers can positively interact in order to facilitate successful ID-POCT implementation.

- ID-POCT specific pre-implementation issues indicate those pre-implementation issues that are particularly important for certain types of ID-POCT and situations.

- Consultation and/or adoption of these guidelines will establish a firm foundation for the successful implementation of ID-POCT within many different types of medical institutions.

- These guidelines may need to be adapted in special circumstances involving: 'low experience', 'extreme' and 'limited resource' environments.

\section{References}

Papers of special note have been highlighted as:

- of interest; $\bullet \bullet$ of considerable interest

1 Luppa PB, Müller C, Schlichtiger A. Point-of-care testing (POCT): current techniques and future perspectives. Trends Anal. Chem. 30(6), 887-898 (2011).

- Outlines some of the technologies behind point-of-care test (POCT) devices.

2 Wienceka J, Nicholsa J. Issues in the practical implementation of POCT: overcoming challenges. Expert Rev. Mol. Diagn. 16(4), 415-422 (2016).
- Outlines POCT implementation quality issues.

3 ISO. Medical laboratories - requirements for quality and competence. http://iso.org/iso/catalogue_detail

4 ISO. Point-of-care testing (POCT) - requirements for quality and competence. http://iso.org/iso/home/store/catalogue_tc

5 CLSI shop. Standards Documents for point-of-care testing. http://shop.clsi.org/point-of-care-documents/

6 UPMC health security. http://upmchealthsecurity.org/our-work/
7 Pai NP, Vadnais C, Denkinger C, Engel N, Pai M. Point-of-care testing for infectious diseases: diversity, complexity, and barriers in low- and middle-income countries. PLoS Med. 9(9), e1001306 (2012).

-• Highlights the specific needs of low- and middle-income countries, which may often be overlooked when designing and implementing infectious disease POCT (ID-POCT).

8 Drancourt M, Michel-Lepage A, Boyer S, Didier R. The point-of-care laboratory in clinical microbiology. Clin. Microbiol. Rev. 29(3), 429-447 (2016). 
- An excellent overview of current ID-POCT issues including key examples of target micro-organisms.

9 Kaman WE, Andrinopoulou E-R, Hays JP. Perceptions of point-of-care infectious disease testing among European medical personnel, point-of-care test kit manufacturers, and the general public. Patient Prefer. Adherence 7, 559-577 (2013).
- Documents the actual need for ID-POCT from different end-user viewpoints.

10 Rapid Microbiology. www.rapidmicrobiology.com

11 Longitude Prize. https://longitudeprize.org

12 European Commission. Better use of antibiotics - $€ 1$ million.

http://ec.europa.eu/research/horizonprize
13 U.S Food \& Drug Administration. Emergency Preparedness. http://fda.gov/EmergencyPreparedness

14 U.S Food \& Drug Administration. Medical Devices.

http://www.fda.gov/MedicalDevices/Safety/ 